\title{
Montreal Cognitive Assessment Naming Test
}

National Cancer Institute

\section{Source}

National Cancer Institute. Montreal Cognitive Assessment Naming Test. NCI Thesaurus.

Code C162710.

In this test three pictures of animals are shown and the individual is asked to name each one. 